\title{
Avaliação da eficiência de caulinita intercalada com dimetilsulfóxido em adsorção com o Zn(II) em meio aquoso - cinética do processo de adsorção
}

\section{(Evaluation of intercaled kaolinite efficiency with dimetilsulfoxide in adsorption with $\mathrm{Zn}(\mathrm{II})$ in aqueous medium - kinetics of the adsorption process)}

\author{
D. L. Guerra', J.A.Sousa ${ }^{2}$, C. Airoldi, R. R.Viana ${ }^{2}$ \\ ${ }^{1}$ Instituto de Química, Universidade Estadual de Campinas, C.P. 6154, Campinas, SP 13083-970 \\ ${ }^{2}$ Centro de Recursos Naturais, Universidade Federal de Mato Grosso, Cuiabá, MT 78060-900 \\ dlguerra@iqm.unicamp.br
}

\begin{abstract}
Resumo
Amostras de caulinita oriundas da região do Rio Capim, estado do Pará, Brasil, foram intercaladas com dimetilsulfóxido - DMSO. As amostras de caulinita naturais e intercaladas foram utilizadas em processo de adsorção com $\mathrm{Zn}$ (II) em meio aquoso em pH 5,0 e temperatura controlada de $298 \pm 1 \mathrm{~K}$. As propriedades físico-químicas das amostras de caulinita foram otimizadas pelo processo de

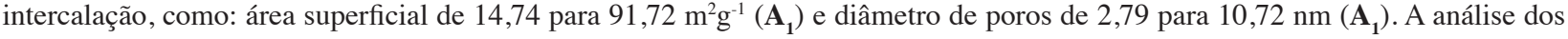
resultados experimentais de adsorção foi feita pelos modelos de Langmuir, Temkin e Freundlich. O modelo de Langmuir apresentou melhor aproximação com os dados experimentais de adsorção. Estes resultados foram bem representados pelo modelo cinético de segunda ordem de Lagergren, com a taxa constante $K_{2}$ no intervalo de $4,76 \times 10^{-3}$ a $11,81 \times 10^{-3} \mathrm{~g}\left(\mathrm{mmol}\right.$.min) ${ }^{-1}\left(\mathbf{A}_{2}\right)$. O processo de adsorção foi considerado rápido alcançando o equilíbrio em 180 min.
\end{abstract}

Palavras-chave: caulinita, adsorção, isotermas, cinética.

\begin{abstract}
The kaolinite clay samples from Capim River region, Pará state, Brazil, were intercalated with dimethylsulfoxide - DMSO. The natural and intercalated kaolinite were used in adsorption process with $\mathrm{Zn}(\mathrm{II})$ in aqueous medium at $\mathrm{pH} 5.0$ and controlled temperature of $298 \pm 1 \mathrm{~K}$. The physical-chemical properties of kaolinite samples were optimized for the intercalation process, such as: specific area of 14.74 to $91.72 \mathrm{~m}^{2} \mathrm{~g}^{-1}\left(\boldsymbol{A}_{1}\right)$ and pore diameter of 2.79 to $10.72 \mathrm{~nm}\left(\boldsymbol{A}_{1}\right)$. The adsorption experimental results were analyzed for Langmuir, Temkin and Freundlich models, the Langmuir model has been presented best approximation with experimental adsorption isotherms data. These results best fitted the second order kinetic of Lagergren model with rate constant $K_{2}$, in the range of $4.76 \times 10^{-3}$ to $11.81 \times 10^{-3} \mathrm{~g}(\mathrm{mmol} . \mathrm{min})^{-1}\left(\boldsymbol{A}_{2}\right)$. The adsorption process was very fast and equilibrium was approached within $180 \mathrm{~min}$.
\end{abstract}

Keywords: kaolinite, adsorption, isotherms, kinetics.

\section{INTRODUÇÃO}

Os materiais lamelares naturais modificados despertam grande interesse no meio científico e tecnológico, estes materiais apresentam características físico-químicas que possibilitam sua utilização nos mais variados setores de atividades. Entre esses materiais, destaca-se o argilomineral caulinita que atualmente é alvo de pesquisas que objetivam produzir novos materiais, através de intercalação de íons, delaminação, hidratação e acidificação com soluções ácidas em baixa concentração [1-5].

Nas ultimas décadas surgiram muitos estudos que objetivaram melhorar estruturalmente as argilas naturais como a atapugita [6], caulinita [7], e montmorilonita [8, 5] com o ancoramento de espécies que atuem dentro da estrutura como novos sítios reativos atribuindo a estes materiais, maior poder reativo e possibilitando que tal material seja aplicado para os mais diversos fins, como por exemplo: em processos de adsorção [9], catálise [10, 11], em nanocompósitos [12,13], na produção de sensores [14, 15] e eletrodos [16, 17], na utilização como ante bactericida no combate a patogênicos [18].

Atividades industriais, aplicação de fertilizantes e deposição inadequada de resíduos levam ao importante acréscimo de metais pesados e outros contaminantes em solos [5]. Por carregamento superficial ou percolação, estes 
metais podem contaminar águas superficiais ou subterrâneas. Dentre os vários métodos de tratamento de contaminantes, como precipitação e troca iônica, a adsorção é um dos mais empregados para a remoção de metais pesados. A argila caulim, cujo principal constituinte é o argilomineral caulinita é um dos minerais mais abundantes em solos e sedimentos. Diversos estudos mostraram que inúmeros argilominerais podem atuar com grande capacidade adsortiva em processos com espécies de aminas como metil-, etil-, butilamina [5] e metais pesados como $\mathrm{Cd}, \mathrm{Pb}, \mathrm{Zn}, \mathrm{Ni}, \mathrm{Cu}$, etc. [19-21], apresentando opções de baixo custo, baixa toxidade e fácil obtenção da superfície adsorvente para aplicação em área com graves problemas de contaminação.

Este trabalho teve como finalidade desenvolver um estudo de modificação físico-química de duas amostras naturais de caulinita, obtidas na região amazônica, através da intercalação com dimetil sulfóxido (DMSO), aplicar os materiais resultantes em processo de adsorção com metal divalente $\mathrm{Zn}$ (II) em meio aquoso com $\mathrm{pH}$ igual a 5,0 e temperatura controlada de $298 \pm 1 \mathrm{~K}$. O desenvolvimento do estudo cinético de adsorção foi realizado através do modelo de pseudo-segunda ordem de Lagergren.

\section{EXPERIMENTAL}

\section{Material original}

As amostras de caulinita foram coletadas na região do estado do Pará (Rio Capim), onde as reservas naturais deste argilomineral são bastante significativas, principalmente devido a fatores climáticos que atuam diretamente em sua formação geológica, como a lixiviação do solo amazônico. As amostras estudadas neste trabalho foram provenientes desse depósito que se localiza a NE do Estado do Pará, entre a rodovia Belém-Brasília (trecho entre Ipixuna do Pará e Mãe do Rio) e o médio curso do Rio Capim.

\section{Preparação das amostras do argilomineral}

Foram pesadas $200 \mathrm{mg}$ da fração do argilomineral de cada amostra em uma balança semi-analítica. As amostras foram dissolvidas em água destilada para $10,0 \mathrm{~cm}^{3} \mathrm{em}$ balões volumétricos. Pequenas alíquotas foram retiradas e colocadas em lâminas de vidro, previamente identificadas, até o seu completo preenchimento. As lâminas orientadas preparadas foram colocadas sobre uma bancada e secas ao ar em temperatura ambiente, durante três dias.

\section{Métodos de caracterização}

A separação granulométrica foi realizada com as amostras devidamente pesadas em balança semi-analítica (Bosch P115), dissolvidas em água destilada e submetidas a um aparelho de ultra-som (Thorton), com o objetivo de provocar a dispersão das partículas e sua homogeneização em meio líquido. Na separação das frações silte e argila, que estavam presentes em solução, as amostras foram tratadas com $\mathrm{Na}_{2} \mathrm{P}_{2} \mathrm{O}_{7}$ para evitar a floculação e depois separadas por centrifugação (Heraeus Christ GMBH osterade), utilizando velocidade de 1000 rpm e tempo de separação da fração silte de 2 min, novamente a fração argila resultante foi centrifugada (2500 rpm e $10 \mathrm{~min}$ ) para a retirada do $\mathrm{Na}_{2} \mathrm{P}_{2} \mathrm{O}_{7}$.

Para a obtenção de difratogramas de raios X (DRX), foi utilizado o método do pó para as amostras naturais e intercaladas de caulinita (Philips PW1050) controlado por um sistema PW 3710, com radiação $\mathrm{k}_{\alpha}$ do cobre (1,54060 Á), monocromador e gerador de tensão $45 \mathrm{kV}$ e corrente $35 \mathrm{~mA} \mathrm{e}$ passo de $1 \mathrm{grau} / \mathrm{min}$.

Para a análise química, as amostras naturais e intercaladas passaram por processo de abertura ácida e foram analisadas pelo método de ICP-OES (Perkin Elmer 3000DV) para os elementos $\mathrm{Na}, \mathrm{K}, \mathrm{Ca}$ e Al; para os elementos $\mathrm{Si}$, Fe e Ti foi utilizado o método de espectroscopia na região do visível em equipamento com duplo-feixe Varian modelo Cary 1E, com celas de $10 \mathrm{~mm}$ de caminho óptico na faixa $360-830 \mathrm{~nm}$.

Os valores da área específica (AE), volume total dos poros (VTP) e diâmetro médio dos poros (DMP) foram obtidos a partir de $249 \mathrm{mg}$ de cada amostra. As amostras foram aquecidas a $423 \mathrm{~K}$ por $1 \mathrm{~h}$ e depois foram resfriadas com nitrogênio líquido a $77 \mathrm{~K}$ em um analisador de adsorção gasosa Quantachrome Nova-1200/5.01.

Para os estudos de microscopia eletrônica de varredura (MEV), as amostras foram preparadas em lâminas orientadas em $298 \pm 1 \mathrm{~K}$ e metalizadas com ouro. O instrumento utilizado foi um LEO-ZEISS $430 \mathrm{Vp}$ em condições de análise utilizando imagens secundárias obtidas a $20 \mathrm{kV}$ com distância de trabalho $11 \mathrm{~mm}$.

\section{Intercalação das amostras de caulinita}

Duas porções de aproximadamente $2 \mathrm{~g}$ de caulinita foram misturadas a $80,0 \mathrm{~cm}^{3}$ de soluções com variadas concentrações de dimetilsulfóxido (DMSO) em água à temperatura ambiente e sob constante agitação mecânica. Após $52 \mathrm{~h}$ de contato, aproximadamente, a reação de intercalação foi encerrada e a cada mistura foi centrifugada. Em seguida, o sobrenadante de cada mistura foi descartado e as amostras de caulinita intercalada foram retiradas do interior dos tubos de centrífuga com o auxílio de uma espátula.

Procedimento e técnica utilizada para a determinação da adsorção de Zn(II)

O processo de adsorção do cátion divalente em solução foi feito utilizando o método de batelada, em que massa de $0,050 \mathrm{~g}$ de caulinita natural e intercalada foi dissolvida em $20,0 \mathrm{~cm}^{3}$ de água deionizada; tal sistema recebeu alíquotas que variaram de 1,00 a $2,00 \mathrm{~cm}^{3}$ de solução de $0,050 \mathrm{~mol}$. $\mathrm{dm}^{-3}$ do cátion, previamente padronizada. A quantificação foi feita através de ICP-OES em um equipamento Perkin Elmer 3000DV, utilizando o sobrenadante de cada ponto. Para a leitura, os sobrenadantes foram diluidos para adequar sua concentração à janela de leitura do equipamento. $\mathrm{O}$ valor de 
cada $\mathrm{N}_{\mathrm{f}}$ foi determinado pela equação $\mathrm{A}$. O modelo utilizado na análise da isoterma construída por cada ponto foi o de Langmuir descrito pela equação B [17]:

$$
\mathrm{N}_{\mathrm{f}}=\left(\mathrm{N}_{\mathrm{ad}}-\mathrm{N}_{\mathrm{s}}\right) / \mathrm{m}
$$

em que $\mathrm{N}_{\mathrm{f}}$ é o número de moles fixos por grama de matriz, $\mathrm{N}_{\mathrm{ad}}$ é o número de moles adicionados inicialmente na matriz, $\mathrm{N}_{\mathrm{s}}$ é o número de moles encontrado no sobrenadante e número máximo de moles na formação da monocamada e m é a massa da matriz utilizada [17].

$$
\left[\mathrm{C}_{\mathrm{s}} / \mathrm{N}_{\mathrm{f}}\right]=\left[1 /\left(\mathrm{K}_{\mathrm{L}} \mathrm{x} b\right)\right]+\left[\mathrm{C}_{\mathrm{s}} / \mathrm{b}\right]
$$

em que $C_{\mathrm{s}}$ é a concentração em mol.dm ${ }^{-3}$ do sobrenadante em equilíbrio e b é uma constante de proporcionalidade que contempla a constante de equilíbrio $\mathrm{K}_{\mathrm{L}}$.

Considerando $\mathrm{C}_{\mathrm{s}} / \mathrm{N}_{\mathrm{f}}$ como variável dependente e $\mathrm{C}_{\mathrm{s}}$ como variável independente, obtêm-se os valores de $\mathrm{K}_{\mathrm{L}} \mathrm{e}$ b, em que $1 / \mathrm{K}_{\mathrm{L}} \mathrm{b}$ é o coeficiente linear e $1 / \mathrm{b}$ é o coeficiente angular da reta.

A equação de Freundlich é a seguinte [5]:

$$
\mathrm{N}_{\mathrm{f}}=K f \mathrm{C}_{\mathrm{s}}{ }^{1 / n}
$$

em que $\mathrm{n}$ indica, quantitativamente, a reatividade dos sítios energéticos do material e $K f$ pode sugerir a adsorção do íon no material. A equação $C$ pode ser escrita na forma:

$$
\log \mathrm{N}_{\mathrm{f}}=\log K f+1 / \mathrm{n} \log \mathrm{C}_{\mathrm{s}}
$$

Fazendo-se o gráfico de $\log \mathrm{N}_{\mathrm{f}}$ versus $\log \mathrm{C}$, obtêm-se os valores de $K f$ e n, onde log $K f$ é o coeficiente linear e 1/n é o coeficiente angular da reta.

A equação de Temkin é a seguinte [5]:

$$
\mathrm{N}_{\mathrm{f}}=\mathrm{A} \ln \mathrm{C}_{\mathrm{s}}+\mathrm{B}
$$

em que A e B são parâmetros da equação. Fazendo-se o gráfico de $\mathrm{N}_{\mathrm{f}}$ versus ln $\mathrm{C}_{\mathrm{s}}$ obtêm-se os valores de $\mathrm{A}$ e $\mathrm{B}$, onde B é o coeficiente linear e A é o coeficiente angular da reta. Os coeficientes obtidos a partir destas equações foram utilizados para ajustar o modelo teórico aos dados experimentais.

\section{RESULTADOS E DISCUSSÃO}

\section{Caracterização das amostras}

Na Tabela I estão apresentados os resultados de análise química das amostras naturais $\left(\mathrm{A}_{1}\right.$ e $\left.\mathrm{A}_{2}\right)$. As amostras apresentaram valores coerentes, no que diz respeito à quantidade de silício, alumínio e o alto teor de titânio, sugerindo a formação de anatásio. Tais resultados foram comparados com os parâmetros reportados da argila teórica [22].

Analisando os resultados de difração de raios $\mathrm{X}$ da amostra natural, $A_{1}$ (Fig. 1), foram constatadas frações dos minerais goethita, hematita, quartzo, sendo caulinita o maior componente; esta conclusão está baseada nos dados obtidos com a análise química e nos difratogramas de raios $\mathrm{X}$ que foram analisados conjuntamente.

Os difratogramas referentes às amostras de caulinita intercaladas apresentaram formação de nova fase em $d_{001}$ $=11,5 \AA\left(\mathrm{A}_{1 \mathrm{DSMO}}\right)$ e $11,4 \AA\left(\mathrm{A}_{2 \mathrm{DSMO}}\right)$, comparando com o espectro obtido com a natural (Fig. 2). Também foi observado que houve mudança quanto à intensidade dos picos, principalmente o pico $\mathrm{d}_{001}$ referente à caulinita; outra observação importante está no fato de que não foi mostrada qualquer alteração na largura e na forma dos picos dos espectros das matrizes intercaladas, sugerindo que não houve comprometimento do grau de cristalinidade das amostras intercaladas em relação às amostras naturais.

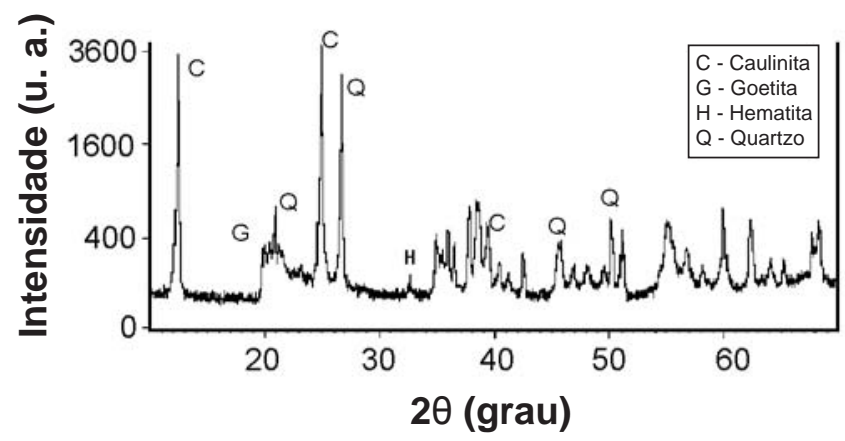

Figura 1: Difratograma de raios $\mathrm{X}$ da amostra de caulinita natural $\left(\mathrm{A}_{1}\right)$.

[Figure 1: X-ray diffraction pattern of natural kaolinite sample $\left(A_{1}\right)$.]

Tabela I - Análise química das matrizes naturais.

[Table I - Chemical analysis of natural matrices.]

\begin{tabular}{ccccccccccccc}
\hline $\begin{array}{c}\text { Constituinte } \\
\text { Amostras }\end{array}$ & $\mathrm{Si}^{4+}$ & $\mathrm{Al}^{3+}$ & $\mathrm{Fe}^{3+}$ & $\mathrm{Ti}^{4+}$ & $\mathrm{P}^{5+}$ & $\mathrm{H}_{2} \mathrm{O}^{+}$ & $\mathrm{H}_{2} \mathrm{O}^{-}$ & $\mathrm{Ca}^{2+}$ & $\mathrm{Mg}^{2+}$ & $\mathrm{Na}^{+}$ & $\mathrm{K}^{+}$ \\
\hline $\mathrm{A}_{1}$ & 45,27 & 39,24 & 0,36 & 0,42 & 0,06 & 14,13 & 0,20 & 0,11 & 0,04 & 0,09 & 0,08 \\
$\mathrm{~A}_{2}$ & 45,12 & 39,02 & 0,65 & 0,81 & 0,02 & 14,05 & - & 0,12 & 0,03 & 0,09 & 0,09 \\
\hline
\end{tabular}




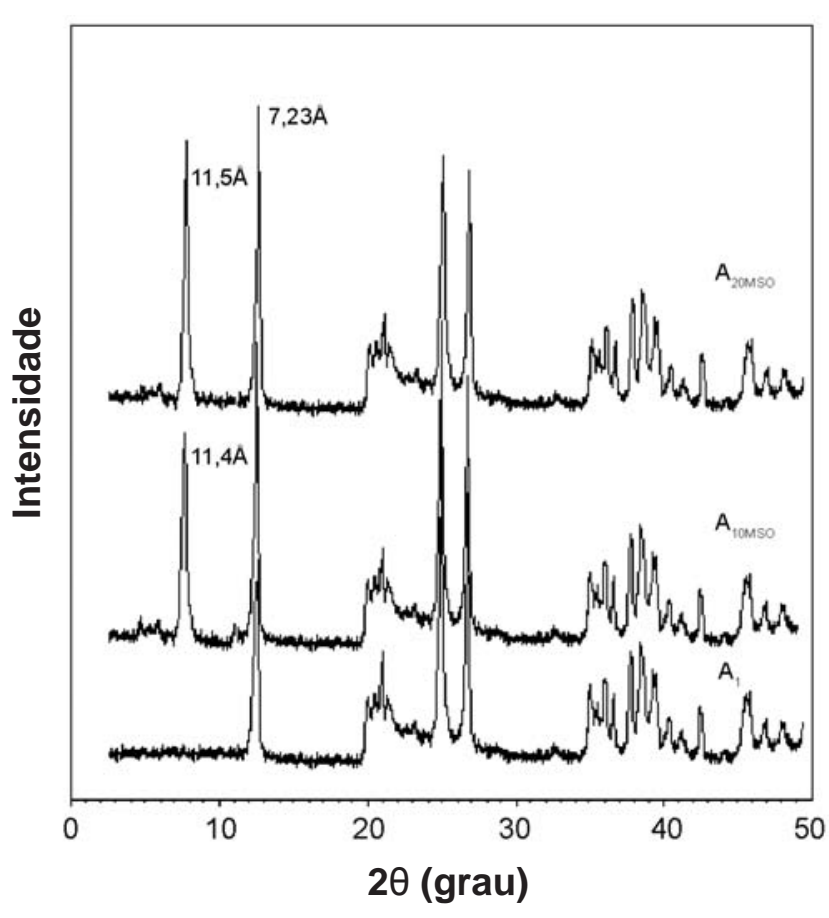

Figura 2: Difratogramas de raios $\mathrm{X}$ das amostras de caulinita intercalada.

[Figure 2: X-ray diffraction patterns of intercalated kaolinite samples.]

Na Tabela II estão apresentados os resultados da avaliação textural para amostras de caulinita naturais e intercaladas. Nas amostras de caulinita natural, a distribuição dos meso e microporos (cálculos pelo método BJH) comportou-se de maneira bimodal e nas amostras de caulinita intercalada, a distribuição apresenta-se de maneira unimodal (Fig. 3); tais resultados estão de acordo com os reportados [19]. As isotermas de adsorção de $\mathrm{N}_{2}$ correspondem às isotermas

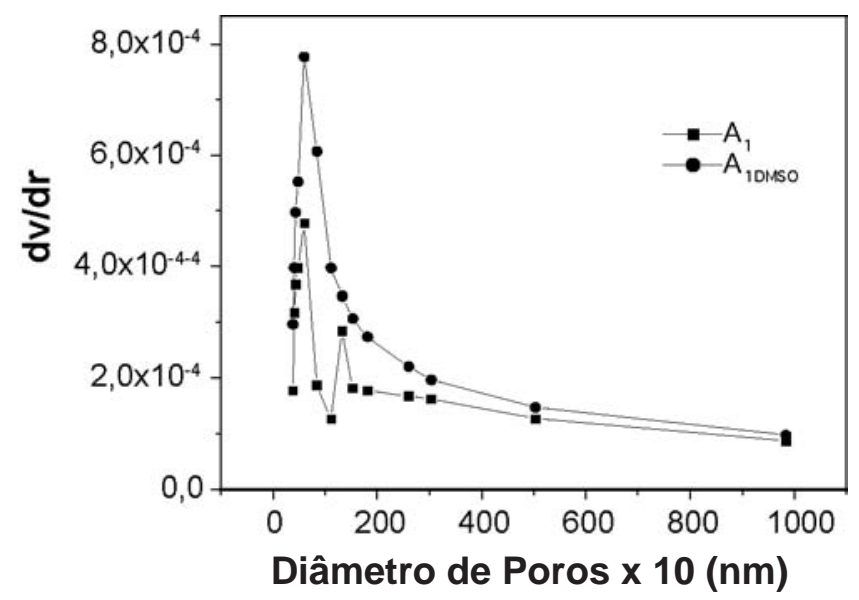

Figura 3: Curva de distribuição de tamanho de poros para amostras de caulinita natural e intercalada.

[Figure 3: Pore size distribution curves of natural and intercalated kaolinite samples.] do tipo IV das amostras naturais e intercaladas segundo a classificação de BDDT (Brunauer, Deming, Deming e Teller), característica de materiais mesoporosos, podendo conter microporos. Verifica-se um aumento considerável na área específica das amostras intercaladas em relação aos valores obtidos para as amostras naturais, aproximadamente 622 e $648 \%$ de crescimento para as amostras $A_{1 \text { DMso }}$ e $\mathrm{A}_{2 \text { DMso }}$, respectivamente. Este resultado é esperado pela presença de DMSO ancorado nas superfícies das amostras de caulinita natural. Outro dado significativo está no diâmetro médio dos poros que apresentou crescimento de $7,9 \mathrm{~nm}\left(\mathrm{~A}_{1}\right)$ comprovando a obtenção de superfície com poros maiores pela funcionalização com DMSO.

A Fig. 4 apresenta micrografias obtidas através de microscopia eletrônica de varredura (MEV). A caulinita pura apresenta cristais pseudo-hexagonais na forma de pilastras e cristais na forma de lamelas. A maioria dos cristais apresenta cantos definidos e ângulos próximos a 90 e $120^{\circ}$, característicos da estrutura da caulinita [6]. Após a intercalação, as amostras de caulinita intercalada apresentaram-se na forma de cristais esfoliados, ligados uns aos outros formando uma espécie de matriz compacta, conseqüência da ação direta do agente intercalante. Na amostra intercalada $\mathrm{A}_{2 \mathrm{DMSO}}$ é observado cristais de menor porte, porém com o formato de cristal com as mesmas características da caulinita intercalada $\mathrm{A}_{1 \mathrm{DSMO}}$, cristais em forma de folha, esta alteração do formato dos cristais, sugere que houve comprometimento da morfologia e tamanho do cristal da caulinita natural com o processo de intercalação.
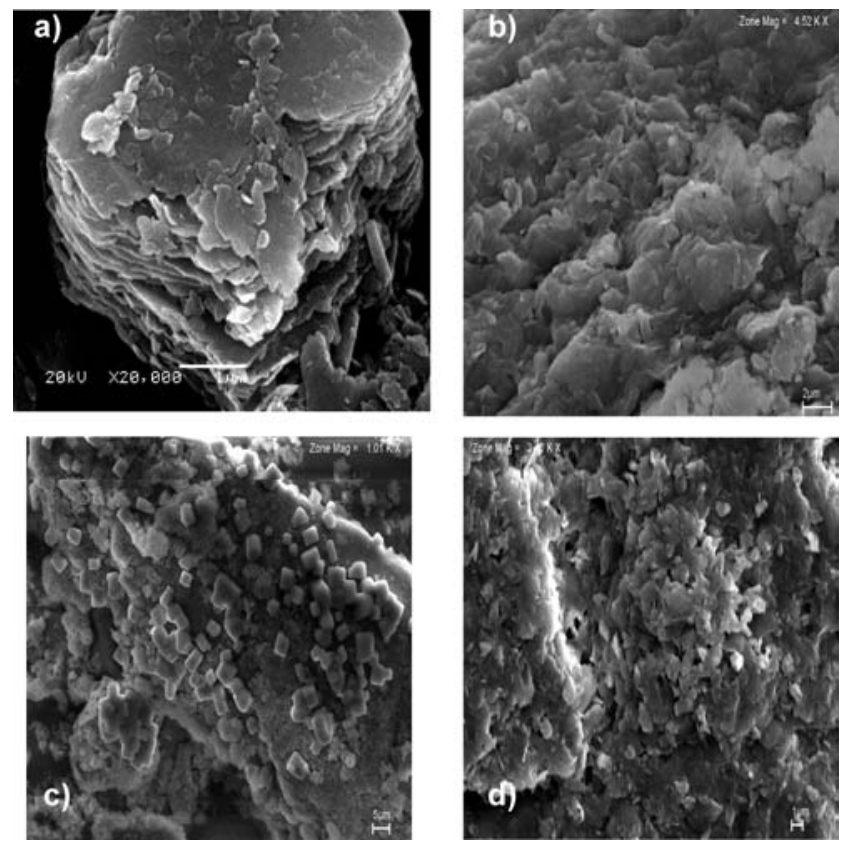

Figura 4: Micrografias obtidas por microscopia eletrônica de varredura das amostras $A_{1}(a), A_{1 \text { DMSO }}(b), A_{2}$ (c), $A_{2 D M S O}(d)$. [Figure 4: SEM micrographs of the samples: $A_{1}(a), A_{1 D M S O}(b), A_{2}$ (c), $\left.A_{2 D M S O}(d).\right]$ 
Tabela II - Resultados da análise textural das amostras naturais e intercaladas.

[Table II - Results of textural analysis of natural and intercalated samples.]

\begin{tabular}{lllll}
\hline Amostra & $\begin{array}{l}\text { Área } \\
\text { específica } \\
\mathrm{BET} \\
\left(\mathrm{m}^{2} \cdot \mathrm{g}^{-1}\right)\end{array}$ & $\begin{array}{l}\text { Aumento } \\
\text { da área } \\
\text { específica } \\
(\%)\end{array}$ & $\begin{array}{l}\text { Vol. total } \\
\text { de poros } \\
\left(\mathrm{cm}^{3} \cdot \mathrm{g}^{-1}\right)\end{array}$ & $\begin{array}{l}\text { Diâmetro } \\
\text { médio } \\
\text { de poros } \\
(\mathrm{nm})\end{array}$ \\
\hline $\mathrm{A}_{1}$ & 14,74 & - & 0,13 & 2,79 \\
$\mathrm{~A}_{2}$ & 15,03 & - & 0,13 & 2,51 \\
$\mathrm{~A}_{1 \text { DMSO }}$ & 91,73 & 622 & 0,07 & 10,72 \\
$\mathrm{~A}_{2 \text { DMSO }}$ & 97,46 & 648 & 0,10 & 9,01 \\
\hline
\end{tabular}

Processo de adsorção com Zn(II)

O processo de adsorção de íons de $\mathrm{Zn}(\mathrm{II})$ acontece com o ancoramento desta espécie nos sítios básicos ativos da caulinita natural. Estes sítios foram produzidos pela descompensação de cargas elétricas que gera a capacidade de troca catiônica (CTC) nas superfícies externas da caulinita, com a intercalação com DMSO, estes sítios são ativados. Outro fato importante que deve ser ressaltado é a exposição das superfícies internas, que são expostas pela entrada de íons $\mathrm{H}^{+}, \mathrm{H}_{2} \mathrm{O}, \mathrm{H}_{3} \mathrm{O}^{+}$e principalmente íons de DMSO entre as lamelas, havendo a ocorrência de novos sítios ácidos aderidos a estas superfícies, que são prováveis receptores de espécies a serem adsorvidas por estas matrizes. Foi observado crescente valor adsorvido de $\mathrm{Zn}$ (II) nas matrizes intercaladas, confirmando a existência de maior número de sítios ativos nestas matrizes (Fig. 5a). A análise das isotermas experimentais foi realizada pelos modelos de Langmuir, Freundlich e Temkin e os valores das constantes encontrados com estas equações estão expostos na Tabela III. O modelo de Langmuir apresentou melhores resultados, havendo aproximação significativa dos resultados obtidos experimentalmente. O desempenho do modelo de Freundlich apresentou aproximação satisfatória para os dados experimentais obtidos em baixa concentração, apresentando limitações em altas concentrações (Fig. 5b). A representação esquemática do processo de adsorção, se dá com o cátion metálico $\left(\mathrm{M}^{2+}\right)$ podendo ser adsorvido pelos sítios reativos presentes na estrutura da caulinita representado por $\equiv \mathrm{SOH}$, em que $\mathrm{S}$ representa átomos de silício ou alumínio nas superfícies internas e externas da caulinita, os sítios reativos
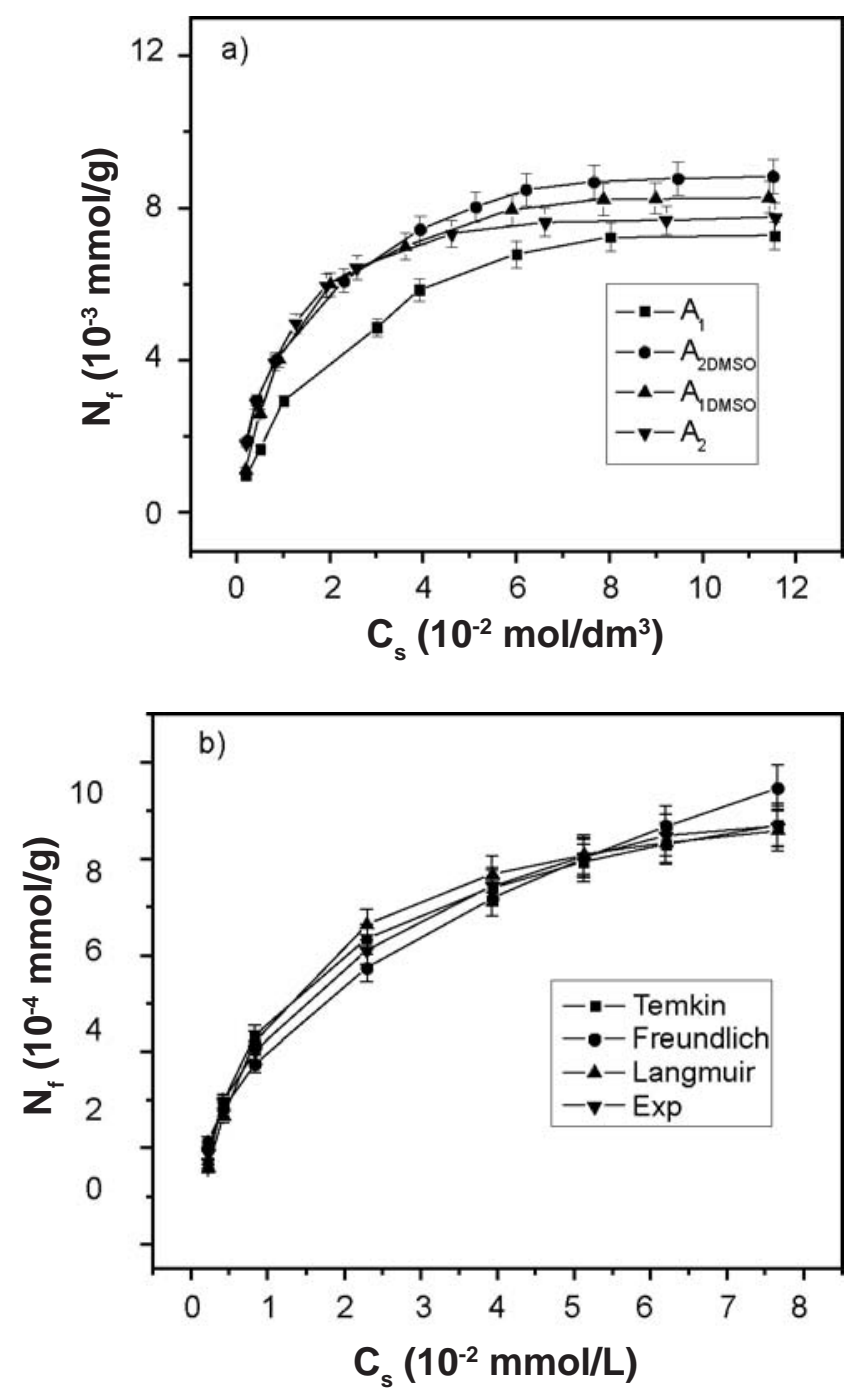

Figura 5: Isotermas de adsorção das amostras naturais e intercaladas com DMSO em adsorção com Zn(II) (a); valores obtidos com as equações de Langmuir, Temkin e Freundlich (b) ( $\mathrm{pH}$ 5,0 e temperatura controlada $=298 \pm 1 \mathrm{~K}$ ).

[Figure 5: Adsorption isotherms of natural and intercalated samples with DMSO in adsorption with $\mathrm{Zn}(\mathrm{II})($ a); values obtained with the Langmuir, Temkin e Freundlich equation (b) (pH 5.0 and temperature controlled $=298 \pm 1 \mathrm{~K})$.]

Tabela III - Constantes de adsorção obtidas com os dados experimentais (pH 5,0 e temperatura controlada $298 \pm 1 \mathrm{~K}$ ). [Table III - Adsorption constants obtained with experimental data ( $p H 5.0$ and controlled temperature $298 \pm 1$ K).]

\begin{tabular}{cccccccccc}
\hline Amostras & \multicolumn{3}{c}{ Langmuir } & \multicolumn{3}{c}{ Freundlich } & \multicolumn{3}{c}{ Temkin } \\
\hline & $b$ & $\mathrm{~K}_{\mathrm{L}}$ & $r^{2}$ & $K f$ & $N$ & $r^{2}$ & $B$ & $A$ & $r^{2}$ \\
\cline { 2 - 10 } $\mathrm{A}_{1}$ & 8,5768 & 1,0372 & 0,999 & 4,2623 & 2,3952 & 0,997 & 1,7228 & 4,6328 & 0,998 \\
$\mathrm{~A}_{2}$ & 8,8309 & 0,9342 & 0,998 & 4,0677 & 2,4099 & 0,998 & 1,9584 & 4,7736 & 0,997 \\
$\mathrm{~A}_{\text {IDMSO }}$ & 10,278 & 9,8905 & 0,999 & 3,4762 & 2,1555 & 0,999 & 1,8596 & 4,3126 & 0,996 \\
$\mathrm{~A}_{\text {2DMSO }}$ & 10,864 & 9,7123 & 0,999 & 3,9312 & 2,3516 & 0,991 & 2,2756 & 4,6417 & 0,997 \\
\hline
\end{tabular}


podem interagir com os cátions de espécies como $\mathrm{Zn}(\mathrm{II})$ em solução, como representado pelas equações abaixo :

$$
\begin{aligned}
& \equiv \mathrm{SOH}+\mathrm{M}^{2+} \rightleftharpoons \equiv \mathrm{SOM}^{+}+\mathrm{H}^{+}, \\
& \equiv \mathrm{SOH}+\mathrm{M}^{2+}+\mathrm{H}_{2} \mathrm{O} \rightleftharpoons \\
& 2 \equiv \mathrm{SOH}+\mathrm{M}^{2+} \rightleftharpoons \mathrm{SOMOH}+2 \mathrm{H}^{+}, \\
& \rightleftharpoons \mathrm{SO}_{2} \mathrm{M}+2 \mathrm{H}^{+} .
\end{aligned}
$$

Sítios situados na superfície com carga negativa $\left(\equiv \mathrm{SO}^{-}\right)$ podem trocar com os cations metálicos $\left(\mathrm{M}^{2+}\right)$, como representados nas Equações I e J.

$$
\begin{aligned}
& 2 \equiv \mathrm{SOH}+\mathrm{M}^{2+} \rightleftharpoons \equiv \mathrm{SO}_{2} \mathrm{M}+2 \mathrm{H}^{+}, \\
& \equiv \mathrm{SOH}+\mathrm{M}^{2+}+\mathrm{H}_{2} \mathrm{O} \rightleftharpoons \equiv \mathrm{SOMOH}+2 \mathrm{H}^{+} .
\end{aligned}
$$

\section{Cinética do processo de adsorção com Zn(II)}

O modelo de Lagergren consiste nas equações de pseudoprimeira ordem e pseudo-segunda ordem. A forma genérica das equações está expressa na equação L [23-25].

$$
\frac{\partial \mathrm{N}_{\mathrm{t}}}{\partial \mathrm{t}}=\mathrm{K}_{1}\left(\mathrm{~N}_{\mathrm{fEQ}}-\mathrm{N}_{\mathrm{f}}\right)
$$

Após a integração da equação $\mathrm{L}$, nas condições $\mathrm{N}_{\mathrm{t}}=$ 0 para $\mathrm{t}=0$ e $\mathrm{N}_{\mathrm{t}}=\mathrm{N}_{\mathrm{t}}$ para $\mathrm{t}=\mathrm{t}$, a forma da equação $\mathrm{L}$ integralizada é

$$
\ln \left(\frac{\mathrm{N}_{\mathrm{fEQ}}}{\mathrm{N}_{\mathrm{f}}}\right)=\ln \mathrm{N}_{\mathrm{fEQ}}-\mathrm{K}_{1} \mathrm{t}
$$

em que $\mathrm{N}_{\mathrm{fEQ}}$ e $\mathrm{N}_{\mathrm{f}}\left(\mathrm{mmol} \mathrm{g}^{-1}\right)$ são concentrações de metal adsorvidas no equilíbrio e concentrações obtidas em isoterma com variação de tempo respectivamente, $\mathrm{k}_{1}$ e a constante de pseudo-primeira-ordem $\left(\mathrm{min}^{-1}\right)$ [23-25].

Através de uma segunda integralização é obtida a equação de pseudo-segunda ordem em que $\mathrm{k}_{2}$ é a constante de pseudo-segunda ordem [23-25].

$$
\frac{\partial \mathrm{N}_{\mathrm{f}}}{\partial \mathrm{t}}=\mathrm{K}_{2}\left(\mathrm{~N}_{\mathrm{fEQ}}-\mathrm{N}_{\mathrm{f}}\right)^{2}
$$

Depois de integralizar a equação nas condições $\mathrm{N}_{\mathrm{t}}=0$ para $t=0$ e $N_{t}=N_{t}$ para $t=t$, a forma integral desta equação é [23-25] :

$$
\mathrm{N}_{\mathrm{f}}=\frac{1}{\frac{1}{\mathrm{k}_{2} \mathrm{~N}_{\mathrm{EQ}}}+\frac{\mathrm{t}}{\mathrm{N}_{\mathrm{EQ}}}}
$$

O aumento da capacidade de adsorção das matrizes intercaladas no equilíbrio é atribuído à exposição das camadas internas, provocada pela presença do íon intercalante na estrutura do argilomineral, que também ocorre pela presença de acessórios com $\mathrm{H}^{+}, \mathrm{H}_{3} \mathrm{O}^{+}$entre as lamelas, provenientes da hidratação do meio intercalante. A adsorção ocorre com o transporte de íons do exterior para o interior dos sítios reativos [2]. A taxa de íons transportados do volume da solução aquosa para a superfície da matriz é um fator que interfere diretamente na cinética do processo de adsorção. Na situação em que uma superfície catalisadora possui maior número de centros reativos, admitindo que tais centros estejam distribuídos em maior número de poros e esses poros com volume considerável, consequentemente esta superfície terá condições de processar maior número de moléculas do adsorbato por unidade de tempo [20, 21]. O tempo de exposição da matriz ao meio aquoso contendo a espécie a ser adsorvida é um fator de grande importância para atingir a condição de equilíbrio químico da reação de
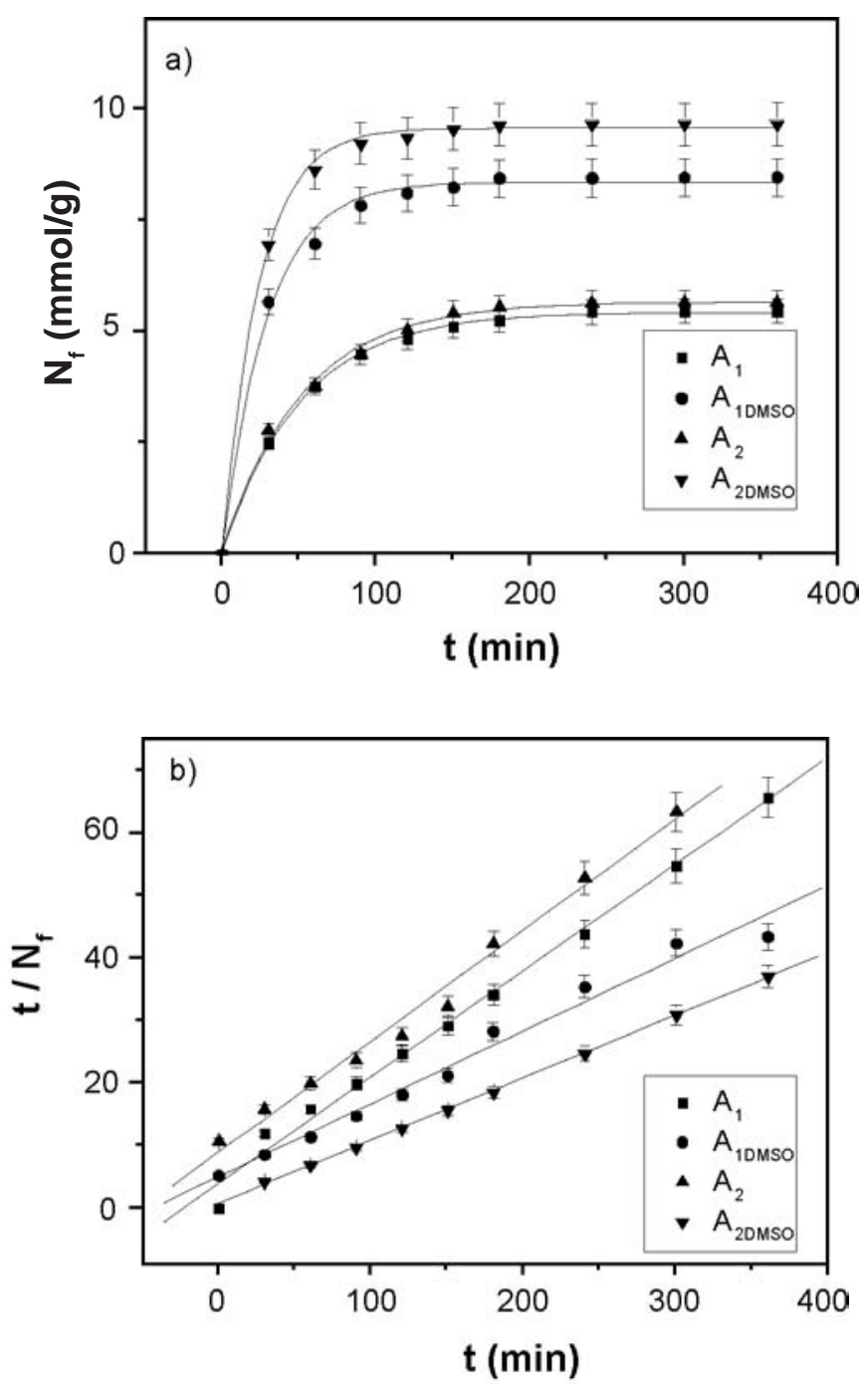

Figura 6: Isotermas de adsorção de tempo das amostras de caulinita em processo de adsorção com o Zn(II) (a); linearização das isotermas (b) (pH 5,0 e temperatura controlada $298 \pm 1 \mathrm{~K}$ ).

[Figure 6: Time isotherms adsorption of kaolinite samples in adsorption process with $\mathrm{Zn}(\mathrm{II})$ (a); linearization of isotherms (b), pH 5.0 and temperature controlled $298 \pm 1$ K.] 
Tabela IV - Parâmetros cinéticos da reação de adsorção das amostras de caulinita naturais e intercaladas ( $\mathrm{pH} \mathrm{5,0}$ temperatura controlada $298 \pm 1 \mathrm{~K}$ ).

[Table IV - Kinetic Parameters of adsorption reaction of samples natural and intercalated (pH 5.0 and controlled temperature $298 \pm 1 \mathrm{~K})$.]

\begin{tabular}{ccccc}
\hline Amostras & \multicolumn{4}{c}{ Cinética de pseudo-segunda ordem } \\
\hline & $\begin{array}{c}K_{2} \times 10^{-3} \\
\mathrm{~g}(\mathrm{mmol} \mathrm{min})^{-1}{ }^{-1}\end{array}$ & $\begin{array}{c}\mathrm{N}_{\mathrm{f}}(\text { Exp }) \\
\left(\mathrm{mmolg}^{-1}\right)\end{array}$ & $\begin{array}{c}\mathrm{N}_{\mathrm{f}}(\mathrm{calc}) \\
\left(\mathrm{mmolg}^{-1}\right)\end{array}$ & $r^{2}$ \\
\hline $\mathrm{A}_{1}$ & 7,3158 & 5,4679 & 5,8603 & 0,9995 \\
$\mathrm{~A}_{2}$ & 4,7570 & 5,6689 & 6,3231 & 0,9981 \\
$\mathrm{~A}_{1 \text { DDMO }}$ & 8,6653 & 8,4789 & 8,8660 & 0,9982 \\
$\mathrm{~A}_{2 \text { DMSO }}$ & 11,8070 & 9,6689 & 9,9601 & 0.9962 \\
\hline
\end{tabular}

adsorção. É observado que a partir de determinado tempo a matriz atinge o equilíbrio do processo de adsorção, sugerindo que os sítios ácidos presentes em toda a sua estrutura, já estejam preenchidos com o ancoramento do adsorbato, tornando-os inertes ou pouco reativos (Fig. 6 e Tabela IV).

\section{CONCLUSÕES}

Fundamentando-se nos satisfatórios ajustes verificados pelo modelo de Langmuir seguido pela equação de Temkin e Freundlich, a equação de Langmuir forneceu melhores resultados na predição de dados de equilíbrio para os sistemas contendo $\mathrm{Zn}$ (II), o que pode ser justificado, pois a equação de Langmuir foi desenvolvida a partir de processos de adsorção de sólidos em solução, sobre adsorventes sólidos com semelhança ao tipo de sistema estudado neste trabalho. A equação de Freundlich apresentou limitações em experimento com maior concentração de Zn(II), com todas as matrizes utilizadas, questão que fica bastante evidenciada quando são observados os últimos pontos das isotermas de concentração. As matrizes intercaladas com DSMO apresentaram grande eficiência na adsorção do Zn(II) comparando os resultados com os obtidos utilizando a matriz na forma natural nas condições de $\mathrm{pH}$ e temperatura apresentados neste trabalho. Os cálculos das constantes cinéticas revelaram uma tendência a um mecanismo físico representado pelo modelo cinético de Lagergrem pseudosegunda ordem. As amostras utilizadas neste estudo quando intercalada com DMSO, provocam uma alteração da cinética do processo; as novas matrizes, com as suas propriedades físico-químicas otimizadas, promovem o crescimento do número de moléculas processadas por unidade de tempo, enfatizando que estas matrizes possuem maior número de sítios reativos em sua estrutura, provenientes da exposição das matrizes naturais ao processo de intercalação.

\section{AGRADECIMENTOS}

Ao CNPq (Proc. 150060/2006-8) eà FAPESP pelo suporte financeiro por meio de bolsas PQ e de pós-doutoramento.

\section{REFERÊNCIAS}

[1] Z. M. Wang, H. Nakajima, E. Manias, T.C. Chung, Macromolecules 36 (2003) 8919.

[2] K. G. Battacharyya, S. S. Gupta, Colloids and Surfaces 277 (2006) 191.

[3] D. M. Manohar, B. F. Noeline, T. S. Anirudhan, Appl. Clay Sci. 31 (2006) 194.

[4] D. L. Guerra, V. P. Lemos, R. S. Angélica, C. Rev. Soc. Port. Mater. 17 (2006) 75.

[5] D. L. Guerra, V. P. Lemos, R. S. Angélica, C. Airoldi, Polyhedron 15 (2006) 2880.

[6] E. Galan, Clay Miner. 31 (1996) 443.

[7] M. Mousty, Appl. Clay Sci. 27 (2004) 159.

[8] S. Y. Lee, R. W. Tank, Appl. Clay Sci. 1 (1985) 145.

[9] P. C. LeBaron, Z. Wang, T. J. Pinnavaia, Appl. Clay Sci. 15 (1999) 11.

[10] F. Cavani, F. Trifiro, A. Vaccari, Catal. Today 11 (1991) 173.

[11] S. R. Chitnis, M. M. Sharma, React. Funct. Polym. 32 (1997) 93

[12] D. Kubies, N. Pantoustier, P. Dubois, A. Rulmont, R. Jerome, Macromolecules 35 (2002) 3318.

[13] M. Cox, J. R. Rus-Romero, T. S. Sheriff, Chem. Eng. J. 84 (2001) 107.

[14] N. Gungor, S. Karaoglan, Mater. Lett. 48 (2001) 168.

[15] M. Darder, M. Colilla, E. Ruiz-Hitzky, Appl. Clay Sci. 28 (2005) 199.

[16] E. Volpert, J. Selb, F. Candau, N. Green, J. F. Argillier, A. Audibert, Langmuir 14 (1998)1870.

[17] I. K. Tonle, E. Ngameni, A. Walcarius, Sens. Actuators B-Chem. 110 (2005) 195.

[18] L. B. Williams, M. Holland, D. D. Eberl, T. Brunet, L. B. De Courrsou, Mineral. Soc. Bull. 139 (2004) 3.

[19] H. Koyuncu, A. R. Kul, N. Yildiz, A. Çalimli, H. Ceylan, J. Hazardous Mater. 141 (2006)128.

[20] S. Olejnik, A. M. Posner, J. P. Quirk, Clay Minerals 8 (1970) 421.

[21] Y. Onal, J. Hazardous Mater. 42 (2006) 1719.

[22] G. Brown, W. G. Brindley, Crystal structures of clay minerals and structures of layer silicates, Mineralogical Soc. Monograph, Cristal structures of clay minerals and their Xray identification, Oxford, 5 (1985) 1.

[23] D. Karadag, Y. Koc, M. Turan, M. Ozturk, J. Hazardous Mater. 144 (2007) 432.

[24] Y. S. Ho, Water Res. 40 (2006) 119.

[25] S. Al-Ashem, Z. Duvnjak, Water Air Soil Pollut. 114 (1999) 251.

(Rec. 07/07/2007, Rev. 25/11/2007) 